\title{
Clinical Guideline Highlights for the Hospitalist: 2019 American Thoracic Society/Infectious Diseases Society of America Update on Community-Acquired Pneumonia
}

\author{
Christopher D Jackson, MD*, Desirée C Burroughs-Ray, MD¹, Nathan A Summers, MD, MSc²
}

\begin{abstract}
'Department of Internal Medicine, University of Tennessee Health Science Center, Memphis, Tennessee; ${ }^{2}$ Division of Infectious Disease, Department of Internal Medicine, University of Tennessee Health Science Center, Memphis, Tennessee.
\end{abstract}

GUIDELINE TITLE: Diagnosis and Treatment of Adults with Community-Acquired Pneumonia: An Official Clinical Practice Guideline of the American Thoracic Society and Infectious Diseases Society of America

RELEASE DATE: October 2019

PRIOR VERSION: 2007 Infectious Diseases Society of America/American Thoracic Society Consensus Guidelines on the Management of Community-Acquired Pneumonia in Adults

DEVELOPER: American Thoracic Society and Infectious Diseases Society of America

FUNDING SOURCE: American Thoracic Society and Infectious Diseases Society of America

TARGET POPULATION: Immunocompetent adult patients with community-acquired pneumonia

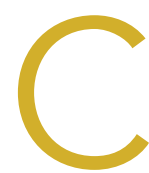

ommunity-acquired pneumonia (CAP) is the second most common cause of hospitalization in the United States, with over 1.5 million unique hospitalizations annually. ${ }^{1}$ CAP is also the most common infectious cause of death in US adults. ${ }^{2}$ The 2019 CAP guideline from the American Thoracic Society (ATS) and the Infectious Diseases Society of America (IDSA) provides recommendations on the diagnosis and management of CAP. The guideline provides 16 recommendations, which we have consolidated to highlight practice changing updates in diagnostic testing, risk stratification, and treatment.

\section{KEY RECOMMENDATIONS}

\section{FOR THE HOSPITALIST}

\section{Diagnostic Testing}

Recommendation 1. In patients with CAP, routine blood cultures, sputum cultures, and urinary antigen tests are not routinely recommended unless severe CAP (Table), history of methicillin-resistant Staphylococcus aureus (MRSA) and/or Pseudomonas infection, or prior hospitalization for which intravenous antibiotics were administered. (Strong recommendation; very low quality of evidence)

The guideline emphasizes that the diagnostic yield of blood/ sputum cultures and urinary antigen testing is low. Additionally, high-quality data showing improved clinical outcomes with routine testing of blood cultures and urinary antigens are lack-

*Corresponding Author: Christopher D Jackson, MD; Email: cjacks67@ uthsc.edu; Telephone: 901-448-5814; Twitter: @ChrisDJacksonMD.

Published online first August 19, 2020.

Received: February 9, 2020; Revised: April 1, 2020; Accepted: April 11, 2020

C 2020 Society of Hospital Medicine DOI 10.12788/jhm.3444 ing. Instead, the guideline suggests obtaining blood cultures, urinary antigens, and sputum gram stain and culture only for patients with severe CAP and those being treated for or having prior infection with MRSA or $P$ aeruginosa. They recommend narrowing therapy as appropriate if cultures are negative for either of these two organisms or previous hospitalization with intravenous antibiotics.

\section{Risk Stratification}

Recommendation 2. In patients with CAP, Pneumonia Severity Index (PSI) or CURB-65 (tool based on confusion, urea level, respiratory rate, blood pressure, and age 65 years or older) scores should not be used to determine general medical ward vs intensive care unit care (ICU). (Strong recommendation; low quality of evidence)

The PSI and CURB-65 scores are not validated to determine location of hospital care. Multiple prognostic models have been studied to predict the need for ICU-level care including SMARTCOP (systolic blood pressure, multilobar chest radiography involvement, albumin level, respiratory rate, tachycardia, confusion, oxygenation, and arterial pH), SCAPA (Study of Community Acquired Pneumonia Aetiology), and the ATS/IDSA criteria (Table). The positive and negative likelihood ratios for needing ICU admission in CAP with either one major or three or more minor ATS/IDSA criteria are 3.28 and 0.21 , respectively.

\section{Treatment}

Recommendation 3a. In patients with nonsevere CAP and no risk factors for MRSA or Pseudomonas infection, empiric treatment with a B-lactam plus macrolide or monotherapy with fluoroquinolones is recommended. (Strong recommendation; high quality of evidence) 
TABLE. ATS/IDSA Criteria for Severe CAP, Defined as One Major Criterion or Three or More Minor Criteria9

\begin{tabular}{ll}
\hline Minor Criteria & Major Criteria \\
\hline Respiratory rate $>30$ breaths/min & Septic shock requiring vasopressors \\
$\mathrm{PaO}_{2} / \mathrm{FiO}_{2}$ ratio $<250$ & Respiratory failure requiring mechanical ventilation \\
$\mathrm{Multilobar}$ infiltrates & \\
Confusion/disorientation & \\
Uremia (blood urea nitrogen level $>20 \mathrm{mg} / \mathrm{dL}$ ) & \\
Leukopenia (white blood cell count $<4,000$ cells/ $/ \mathrm{L}$ ) & \\
Thrombocytopenia (platelet count $<100,000 / \mu \mathrm{L})$ & \\
Hypothermia (core temperature $<36^{\circ} \mathrm{C}$ ) \\
Hypotension requiring aggressive fluid resuscitation
\end{tabular}

Reprinted with permission of the American Thoracic Society. Copyright @ 2020 American Thoracic Society. All right reserved.

Recommendation $3 \mathrm{~b}$. In patients with severe CAP and no risk factors for MRSA or Pseudomonas infection, empiric treatment with a B-lactam plus either a macrolide or fluoroquinolone is recommended. (Strong recommendation; low to moderate quality of evidence)

Microbiologic risk assessment is critical. Risk factors for MRSA or $P$ aeruginosa pneumonia include isolation of these agents in culture and recent hospitalization with receipt of parenteral antibiotics. B-lactam monotherapy is not recommended because previous randomized clinical trials (RCTs) demonstrated inferiority of B-lactam monotherapy to combination therapy for resolution of CAP. The recommended combination therapy for patients with severe CAP without risk factors for MRSA or $P$ aeruginosa infection is a B-lactam plus either a macrolide or a respiratory fluoroquinolone.

Recommendation 4. In patients with suspected aspiration pneumonia, additional anaerobic coverage is not routinely recommended. (Conditional recommendation; very low quality of evidence)

Aspiration often causes a self-limited pneumonitis that will resolve in 24 to 48 hours with supportive care. Use of additional anaerobic coverage in these patients increases risk for complications (eg, Clostridioides difficile infection) without improving outcomes.

Recommendation 5. In patients with nonsevere CAP, corticosteroids are not routinely recommended. (Conditional recommendation; moderate quality of evidence)

There is no direct evidence that steroids reduce mortality or organ failure in nonsevere CAP. Additionally, the use of steroids in CAP can come with considerable risks (eg, secondary infection, hyperglycemia).

Recommendation 6. In hospitalized patients with CAP, empiric coverage for MRSA or $P$ aeruginosa should be limited to patients meeting specific criteria. (Strong recommendation; moderate quality of evidence)

The guideline highlights the current overuse of extended spectrum antibiotics in patients meeting the previous definition of healthcare-associated pneumonia (HCAP). HCAP was defined by the presence of new chest $x$-ray infiltrates in patients with various exposures to healthcare settings (eg, chron- ic dialysis, infusion centers, emergency rooms). Antimicrobial therapy covering MRSA or $P$ aeruginosa should be reserved for patients at risk for MRSA or $P$ aeruginosa infection unless microbiologic testing is negative. Empiric antibiotic selection should incorporate local resistance patterns guided by hospital antibiograms.

Recommendation 7. In adults with CAP, antibiotics should be continued for no less than 5 days with documented clinical stability. (Strong recommendation; moderate quality of evidence)

Hospitalists often determine the length of antibiotic therapy for CAP. Recent studies show extended antibiotic treatment for pneumonia increases risk for adverse events without improving outcomes. Studies also demonstrate patients who receive 5 days of antibiotics total after achieving clinical stability by day 3 do no worse than patients receiving 8 or more days of antibiotics.

\section{CRITIQUE}

This guideline was created by a panel of pulmonologists, infectious disease specialists, general internists, and methodologists using the GRADE (Grading of Recommendations Assessment, Development and Evaluations) approach to draft recommendations. Conflicts of interest were disclosed by all panel members according to the ATS and IDSA policies, and ultimately, two panel members recused themselves owing to conflicts of interest. The inclusion of a large number of RCTs, observational studies, and meta-analyses provides for good generalizability of the guideline published by this group.

Equal support was given in the guideline to all B-lactams listed, including ampicillin/sulbactam, cefotaxime, ceftriaxone, and ceftaroline, regardless of MRSA risk factors. As the authors explicitly state in the guideline, one of the major reasons for abandoning the HCAP classification was to correct the overuse of anti-MRSA and antipseudomonal therapy. ${ }^{3}$ It is surprising, then, that the authors would include ceftaroline, a broad-spectrum cephalosporin that covers MRSA, as first-line therapy for patients without risk factors for MRSA.

The guideline also supported the use of a respiratory fluoroquinolone or a B-lactam with macrolide equally. Although most RCTs have found equal efficacy between these two regimens, ${ }^{4}$ 
there is growing concern about the safety of fluoroquinolones. ${ }^{5}$ While the authors do encourage clinicians to consider these side effects in the main body of the text, a stronger statement could have been made more prominently to warn clinicians of safety concerns with fluoroquinolones.

Finally, while monotherapy with a B-lactam was supported for the treatment of nonsevere outpatient CAP, it was not included in the recommendations for the treatment of hospitalized patients. There is conflicting data on this topic. One RCT failed to show noninferiority of monotherapy, but this was most pronounced among patients with severe pneumonia (PSI category IV) or cases with proven atypical infections. ${ }^{6}$ Another RCT found monotherapy to be noninferior to combination therapy for hospitalized patients not admitted to the ICU.? There is also evidence suggesting that many patients hospitalized with pneumonia have viral rather than bacterial infections, ${ }^{8}$ which brings into question the need for antibiotics in this subset entirely. When these findings are considered from a stewardship perspective and patient safety profile, monotherapy with a B-lactam for hospitalized patients without severe pneumonia could have been considered.

\section{AREAS IN NEED OF FUTURE STUDY}

Future research should track the effects of this guideline's recommendation to narrow empiric therapy on patients empirically treated for MRSA or $P$ aeruginosa infection once sputum and blood cultures are negative, particularly with respect to reduction of time on broad spectrum antimicrobials and clinical outcomes. Similarly, better definitions of which patients require empiric MRSA and $P$ aeruginosa antimicrobial coverage are needed. Ideally, further research will facilitate rapid, cost- effective, and individualized therapy, particularly with growing concerns for antimicrobial resistance and safety.

Disclosures: The authors have no relevant financial conflicts of interest to disclose.

\section{References}

1. Ramirez JA, Wiemken TL, Peyrani P, et al. Adults hospitalized with pneumonia in the United States: incidence, epidemiology, and mortality. Clin Infect Dis. 2017;65(11):1806-1812. https://doi.org/10.1093/cid/cix647

2. Xu J, Murphy SL, Kochanek KD, Bastian BA. Deaths: final data for 2013. Natl Vital Stat Rep. 2016;64(2):1-119.

3. Jones $B E$, Jones $M M$, Huttner $B$, et al. Trends in antibiotic use and nosocomial pathogens in hospitalized veterans with pneumonia at 128 medical centers, 2006-2010. Clin Infect Dis. 2015;61(9):1403-1410. https://doi. org/10.1093/cid/civ629

4. Fogarty C, Siami G, Kohler R, et al. Multicenter, open-label, randomized study to compare the safety and efficacy of levofloxacin versus ceftriaxone sodium and erythromycin followed by clarithromycin and amoxicillin-clavulanate in the treatment of serious community-acquired pneumonia in adults. Clin Infect Dis. 2004;38(Suppl 1):S16-S23.

5. U.S. Food and Drug Administration. Fluoroquinolone antimicrobial drugs information. Accessed February 4, 2020. https://www.fda.gov/Drugs/DrugSafety/InformationbyDrugClass/ucm346750.htm

6. Garin N, Genné D, Carballo S, et al. B-Lactam monotherapy vs B-lactam-macrolide combination treatment in moderately severe communityacquired pneumonia: a randomized noninferiority trial. JAMA Intern Med. 2014;174(12):1894-1901. https://doi.org/10.1001/jamainternmed.2014.4887

7. Postma DF, van Werkhoven $\mathrm{CH}$, van Elden LJ, et al. Antibiotic treatment strategies for community-acquired pneumonia in adults. N Engl J Med. 2015;372(14):1312-1323. https://doi.org/10.1056/nejmoa1406330

8. Jain S, Self WH, Wunderink RG, et al. Community-acquired pneumonia requiring hospitalization among U.S. adults. N Engl J Med. 2015;373(5):415427. https://doi.org/10.1056/nejmoa1500245

9. Metlay JP, Waterer GW, Long AC, et al. Diagnosis and treatment of adults with community-acquired pneumonia: an official clinical practice guideline of the American Thoracic Society and Infectious Diseases Society of America. Am J Respir Crit Care Med. 2019;200(7):e45-e67. https://doi.org/10.1164/ rccm.201908-1581st 